\title{
Benchmarking hybrid assemblies of Giardia and prediction of widespread intra-isolate structural variation
}

Stephen M. J. Pollo ${ }^{1,2}$, Sarah J. Reiling 3 , Janneke Wit' ${ }^{2,4}$, Matthew L. Workentine ${ }^{1}$, Rebecca A. Guy ${ }^{5}$, G. William Batoff ${ }^{6}$, Janet Yee ${ }^{6}$, Brent R. Dixon ${ }^{3}$ and James D. Wasmuth ${ }^{1,2^{*}}$

\begin{abstract}
Background: Currently available short read genome assemblies of the tetraploid protozoan parasite Giardia intestinalis are highly fragmented, highlighting the need for improved genome assemblies at a reasonable cost. Long nanopore reads are well suited to resolve repetitive genomic regions resulting in better quality assemblies of eukaryotic genomes. Subsequent addition of highly accurate short reads to long-read assemblies further improves assembly quality. Using this hybrid approach, we assembled genomes for three Giardia isolates, two with published assemblies and one novel, to evaluate the improvement in genome quality gained from long reads. We then used the long reads to predict structural variants to examine this previously unexplored source of genetic variation in Giardia.
\end{abstract}

Methods: With MinION reads for each isolate, we assembled genomes using several assemblers specializing in long reads. Assembly metrics, gene finding, and whole genome alignments to the reference genomes enabled direct comparison to evaluate the performance of the nanopore reads. Further improvements from adding Illumina reads to the long-read assemblies were evaluated using gene finding. Structural variants were predicted from alignments of the long reads to the best hybrid genome for each isolate and enrichment of key genes was analyzed using random genome sampling and calculation of percentiles to find thresholds of significance.

Results: Our hybrid assembly method generated reference quality genomes for each isolate. Consistent with previous findings based on SNPs, examination of heterozygosity using the structural variants found that Giardia BGS was considerably more heterozygous than the other isolates that are from Assemblage A. Further, each isolate was shown to contain structural variant regions enriched for variant-specific surface proteins, a key class of virulence factor in Giardia.

Conclusions: The ability to generate reference quality genomes from a single MinION run and a multiplexed MiSeq run enables future large-scale comparative genomic studies within the genus Giardia. Further, prediction of structural variants from long reads allows for more in-depth analyses of major sources of genetic variation within and between Giardia isolates that could have effects on both pathogenicity and host range.

Keywords: Long-read sequencing, MinION, Giardia, Structural variants, Heterozygosity, Parasite, Polyploidy, Tetraploid, Genome assembly

*Correspondence: jwasmuth@ucalgary.ca

1 Department of Ecosystem and Public Health, Faculty of Veterinary

Medicine, University of Calgary, Calgary, AB, Canada

Full list of author information is available at the end of the article

c) The Author(s) 2020. This article is licensed under a Creative Commons Attribution 4.0 International License, which permits use, sharing, adaptation, distribution and reproduction in any medium or format, as long as you give appropriate credit to the original author(s) and the source, provide a link to the Creative Commons licence, and indicate if changes were made. The images or other third party material in this article are included in the article's Creative Commons licence, unless indicated otherwise in a credit line to the material. If material is not included in the article's Creative Commons licence and your intended use is not permitted by statutory regulation or exceeds the permitted use, you will need to obtain permission directly from the copyright holder. To view a copy of this licence, visit http://creativeco mmons.org/licenses/by/4.0/. The Creative Commons Public Domain Dedication waiver (http://creativecommons.org/publicdomain/ zero/1.0/) applies to the data made available in this article, unless otherwise stated in a credit line to the data. 


\section{Background}

Giardia intestinalis (syns Giardia lamblia and Giardia duodenalis) is a single-celled, eukaryotic, food- and water-borne intestinal parasite that infects roughly 200 million people worldwide [1]. Infections can cause nausea, vomiting, diarrhea, and impaired growth and cognitive development [1]. The species G. intestinalis includes eight subtypes, named Assemblages A through $\mathrm{H}$, at least two of which are known to infect humans (A and B) [1]. The cells have two diploid nuclei each containing five chromosome pairs [2]. The haploid genome size is $\sim 12.8 \mathrm{Mb}$ [3]. Genome comparisons amongst assemblages of G. intestinalis found only $77 \%$ nucleotide and $78 \%$ amino acid identity in coding regions, suggesting the assemblages may represent different species [4]. Six isolates of G. intestinalis have reference genomes available [3].

Currently, whole genomes are sequenced using secondgeneration technologies, third-generation technologies, or strategies involving combinations of technologies [5-7]. Second-generation sequencing platforms produce high quality reads with low error rates $(0.1 \%$ for Illumina HiSeq) but short lengths (mean length $<250$ bp for Illumina HiSeq), which pose challenges for assembly programs resulting in more fragmented assemblies [8]. In contrast, third-generation sequencing platforms produce much longer reads (mean length $<10,000 \mathrm{bp}$ for PacBio and MinION) but have higher error rates $(10-15 \%$ for PacBio and $>10 \%$ for MinION depending on the chemistry) $[8-10]$. These longer reads have the potential to resolve many genomic areas that are problematic for second-generation data, such as repetitive and/or duplicated regions [10]. Importantly, eukaryotic genomes have many such repetitive and duplicated regions (as much as two- thirds of the human genome may be repetitive elements [11]), making eukaryotic genomes especially good candidates for sequencing with third-generation technologies. Moreover, third-generation data are well suited for examining structural variants within a genome. In diploid and polyploid organisms, the different copies of each chromosome can contain large-scale differences relative to the consensus sequence that is generated during genome assembly, including insertions, deletions, duplications and translocations, in addition to variation at the single nucleotide level (SNPs). Polymorphisms greater than 100 bp are collectively called structural variants. They are a major source of genetic variation, thought to play a larger role in phenotypic variation than SNPs, but are difficult to resolve using second-generation data [12-14]. The tetraploidy of Giardia trophozoites further complicates short-read genome assembly and structural variant detection methods because of the increased computational complexity of constructing four haplotypes for each locus. For a review on the challenges associated with polyploid eukaryotic genomes see [15]. Our expectation is that long-read methods can detect and resolve the potentially three overlapping alternate alleles at any given locus.

The Oxford Nanopore Technologies (ONT) MinION is a third-generation sequencing platform based on nanopore technology $[10,16]$. Briefly, the nucleic acids to be sequenced are driven through small pores in a membrane by an electrical current which causes fluctuations in the current in the pore [10]. Sensors measure these fluctuations, sending the data to a connected computer for processing and storage [10]. Assembling genomes de novo from MinION data involves basecalling of the squiggle files produced by the MinION during sequencing, assembly of the long reads into draft genomes, and polishing of the assemblies.

Here we have generated MinION and Illumina sequence data for G. intestinalis Assemblage A isolate WB (hereafter referred to as Giardia AWB), G. intestinalis Assemblage B isolate GS (hereafter referred to as Giardia BGS), and G. intestinalis isolated from a beaver (hereafter referred to as Giardia beaver). For each isolate, we assembled genomes from the long reads using several long-read assembler programs and evaluated each assembly on the basis of assembly metrics, gene finding, and comparison to the reference genome using whole genome alignments. We then added the short reads to the long-read assemblies to generate hybrid assemblies that were reference quality. After generating reference quality assemblies with the long and short reads, the long reads produced here were then used to investigate heterozygosity in each isolate by detecting the structural variants in each genome.

\section{Methods}

\section{Giardia intestinalis isolates}

Giardia AWB (ATCC 30957) and Giardia BGS (ATCC 50580) were obtained from the American Tissue Culture Collection, while Giardia beaver was a gift from Dr. Gaetan Faubert from McGill University, Canada. Giardia trophozoites were grown in TYI-S-33 medium [17] in 16 -ml screw-capped glass tubes incubated at $37^{\circ} \mathrm{C}$.

\section{DNA extraction}

Ten 16-ml culture tubes of each Giardia isolate (AWB, BGS and beaver) grown to late logarithm stage $\left(\sim 5-8 \times 10^{5}\right.$ cells $\left./ \mathrm{ml}\right)$ were used for genomic DNA isolation. The culture tubes were chilled on ice for $5 \mathrm{~min}$ and the cells were collected by centrifugation at $1100 \times g$ for 15 min at $4{ }^{\circ} \mathrm{C}$. Genomic DNA was extracted with DNAzol Reagent (Thermo Fisher Scientific, Waltham, USA) by 
following the manufacturer's instructions. Briefly, each cell pellet was resuspended and lysed in DNAzol Reagent by gentle pipetting followed by a freeze $(30 \mathrm{~min}$ at $-80{ }^{\circ} \mathrm{C}$ ) and thaw (10 min at room temperature) step. The lysate was then centrifuged at $10,000 \times g$ for $10 \mathrm{~min}$ at $4{ }^{\circ} \mathrm{C}$ to remove insoluble cell debris. The supernatant was transferred to a new tube and the DNA was recovered by centrifugation of the supernatant at $4000 \times g$ for $5 \mathrm{~min}$ at $4{ }^{\circ} \mathrm{C}$. The DNA pellet was washed twice with $75 \%$ ethanol then air-dried. The DNA was resuspended initially in $8 \mathrm{mM} \mathrm{NaOH}$ then neutralized by addition of HEPES to a final concentration of $9 \mathrm{mM}$.

RNA was removed from the DNA sample by the addition of $1-2 \mu \mathrm{l}$ of $20 \mu \mathrm{g} / \mu \mathrm{l}$ RNase A (BioShop, Burlington, Canada) followed by incubation at $65^{\circ} \mathrm{C}$ for $10 \mathrm{~min}$. The degraded RNA was precipitated by the addition of ammonium acetate, incubation at $4{ }^{\circ} \mathrm{C}$ for $20 \mathrm{~min}$ and centrifugation at $12,000 \times g$ for $30 \mathrm{~min}$ at $4{ }^{\circ} \mathrm{C}$. The supernatant was transferred to a new tube and the DNA was precipitated by the addition of $95 \%$ ethanol, incubation at room temperature for $5 \mathrm{~min}$ and centrifugation at $12,000 \times g$ for $20 \mathrm{~min}$ at $4{ }^{\circ} \mathrm{C}$. The DNA pellet was washed once with $0.01 \mathrm{M}$ ammonium acetate in $75 \%$ ethanol and once with $75 \%$ ethanol alone. The DNA pellet was airdried before resuspension in TE buffer $(10 \mathrm{mM}$ Tris- $\mathrm{HCl}$ pH 8.0, 1 mM EDTA).

\section{MinION sequencing}

The 1Dsq library preparation kit SQK-LSK308 was used as recommended by the manufacturer (Oxford Nanopore Technologies, Oxford, UK). Approximately 200 ng of prepared library was loaded onto a FLO-MIN107 (R9.5) flow cell. Data collection was carried out with live basecalling for $48 \mathrm{~h}$, or until no more strands were being sequenced. All sequences were deposited in the sequence read archive (SRA) under accession number PRJNA561185.

\section{Illumina sequencing}

Libraries were prepared using NexteraXT and pairedend sequenced on the MiSeq (v3, $2 \times 300$ cycles) or iSeq 100 (I1, $2 \times 150$ cycles) platforms according to manufacturer instructions (Illumina Inc., San Diego, USA). All sequences were deposited in the SRA under accession number PRJNA561185.

\section{Long-read basecalling, de novo assembly and genome polishing}

Basecalling of all MinION output files was performed with the program Albacore (version 2.0.2) [18] using the full_1dsq_basecaller.py method to basecall both 1D and 1Dsq reads. The flowcell and kit parameters were FLOMIN107 and SQK-LSK308, respectively.
De novo assemblies were performed using the programs Abruijn (version 2.1b) [19], Canu (version 1.6) [20] and SMARTdenovo (version 1.11 running under Perl version 5.22.0) [21]. Abruijn assemblies were conducted using the nanopore platform setting, coverage estimates calculated as the number of bases in the input reads divided by the reference genome size (Table 1) all rounded to the nearest integer, and all other default settings (one polishing iteration, automatic detection of kmer size, minimum required overlap between reads of $5000 \mathrm{bp}$, automatic detection of minimum required kmer coverage, automatic detection of maximum allowed kmer coverage). Canu assemblies were performed using Canu's settings for uncorrected nanopore reads (-nanoporeraw), genome sizes estimated from the reference genome sizes (Table 1), and setting gnuplotTested=true to bypass html output report construction. SMARTdenovo assemblies were conducted using default settings (kmer length for overlapping of 16 and minimum required read length of 5000 bases).

Genome polishing is an error correction step performed on assemblies generated from third-generation data to compensate for the high error rate of the reads [10]. It involves re-evaluating the base calls from the MinION squiggle files together with the read overlap information from the assembly to improve base accuracy and correct small insertions and deletions [22]. Here, polishing was performed with the program Nanopolish (version 0.8.5) following the directions for "computing a new consensus sequence for a draft assembly" [23]. Briefly, the draft genome was first indexed using BWA (version 0.7.15-r1140) [24] and the basecalled reads were aligned to the draft genome using BWA. SAMtools (version 1.6 using htslib 1.6) [25] was then used to sort and index the alignment. Nanopolish then computed the new consensus sequence in $50 \mathrm{~kb}$ blocks in parallel, which were then merged into the polished assembly.

The commands used in the assembling and subsequent analyses can be found in Additional file 1: Text S1.

\section{Read error profile analysis}

Read error profiles were examined for the six Giardia AWB and Giardia BGS runs using the program NanoOK (version v1.31) [26]. NanoOK extracts fasta sequences from the fast 5 files produced by the MinION and aligns them to the reference genome using the LAST aligner (version 876) [27]. It then calculates error profiles for each set of reads that aligned to each contig in the reference. To obtain overall values for all reads in the sequencing run, for each error metric the value for each contig was extracted from the tex file produced by NanoOK and multiplied by the proportion of the total reads mapping to that contig. These values were then summed to yield 
the metric value with respect to all reads in the sequencing run. The sums were scaled according to the proportion of the total reads that were included in the metric calculation (those that were mapped to the contigs) to yield the metric value for all reads used in the analysis.

\section{Long-read assembly evaluation}

The effects on final assembly quality were evaluated for the following parameters: 1D vs 1Dsq input reads, pooling reads for the same organism from multiple runs, assembly program, and number of genome polishing iterations. First, 13 distinct input combinations, that represent all permutations of pooling runs for the same organism for both 1D and 1Dsq reads, were used for de novo assemblies: AWB_0157 1D reads; AWB_0157 1Dsq reads; AWB_0150_0157 1D reads; AWB_0150_0157 1Dsq reads; AWB_2338 1D reads; AWB_2338 1Dsq reads; AWB_2331_2338 1D reads; AWB_0150_0157_2331_2338 1D reads; AWB_0150_0157_2338 1Dsq reads; BGS_2244 1D reads; BGS_2244 1Dsq reads; BGS_2237_2244 1D reads; and BGS_2237_2244 1Dsq reads (Table 1). Each of these input combinations was used to perform a de novo

Table 1 MinION sequencing run metadata, Albacore [18] basecalling results for both 1D and 1Dsq basecalling and read statistics

\begin{tabular}{|c|c|c|c|c|c|c|c|c|}
\hline $\begin{array}{l}\text { Name } \\
\text { used in this } \\
\text { document }\end{array}$ & AWB_0150 & AWB_0157 & AWB_2331 & AWB_2338 & Beaver_2302 & Beaver_2309 & BGS_2237 & BGS_2244 \\
\hline Run name & SRRun1 & SRRun1 & SRRun2 & SRRun2 & SRRun3 & SRRun3 & SRRun4 & SRRun4 \\
\hline Run ID & $\begin{array}{l}\text { 20170720_0150_ } \\
\text { Giardi- } \\
\text { aWB_20170719 }\end{array}$ & $\begin{array}{l}\text { 20170720_0157_ } \\
\text { Giardi- } \\
\text { aWB_20170719 }\end{array}$ & $\begin{array}{l}\text { 20170721_2331_ } \\
\text { Giardi- } \\
\text { aWB_20170721 }\end{array}$ & $\begin{array}{l}\text { 20170721_2338_ } \\
\text { Giardi- } \\
\text { aWB_20170721 }\end{array}$ & $\begin{array}{l}\text { 20170726_2302__ } \\
\text { GiardiaBea- } \\
\text { ver_20170726 }\end{array}$ & $\begin{array}{l}\text { 20170726_2309_ } \\
\text { GiardiaBea- } \\
\text { ver_20170726 }\end{array}$ & $\begin{array}{l}\text { 20170731_2237_- } \\
\text { Giardi- } \\
\text { aGS_20170731 }\end{array}$ & $\begin{array}{l}\text { 20170731_2244_ } \\
\text { Giardi- } \\
\text { aGS_20170731 }\end{array}$ \\
\hline Isolate & Giardia AWB & Giardia AWB & Giardia AWB & Giardia AWB & Giardia beaver & Giardia beaver & Giardia BGS & Giardia BGS \\
\hline $\begin{array}{l}\text { Reference } \\
\text { genome } \\
\text { size (bp) }\end{array}$ & $12,827,416$ & $12,827,416$ & $12,827,416$ & $12,827,416$ & na & na & $11,001,532$ & $11,001,532$ \\
\hline $\begin{array}{l}\text { Sequencing } \\
\text { depth (X } \\
\text { genome } \\
\text { size) }\end{array}$ & 0.5 & 184.6 & 0.1 & 9.9 & 0.7 & 246.8 & 0.9 & 757.6 \\
\hline $\begin{array}{r}\text { Total no. of } \\
1 \mathrm{D} \text { reads }\end{array}$ & 1225 & 329,039 & 237 & 19,531 & 1668 & 382,740 & 1508 & 885,046 \\
\hline $\begin{array}{l}\text { No. of } 1 \mathrm{D} \\
\text { reads } \\
\text { pass }\end{array}$ & 1207 & 304,219 & 152 & 15,842 & 1603 & 354,581 & 1449 & 804,942 \\
\hline $\begin{array}{l}\text { No. of } 1 \mathrm{D} \\
\text { reads fail }\end{array}$ & 18 & 24,820 & 85 & 3689 & 65 & 28,159 & 59 & 80,104 \\
\hline $\begin{array}{l}\text { Percent of } \\
\text { 1D reads } \\
\text { passing }\end{array}$ & 98.5 & 92.5 & 64.1 & 81.1 & 96.1 & 92.6 & 96.1 & 90.9 \\
\hline $\begin{array}{l}\text { Total no. } \\
\text { of IDsq } \\
\text { reads }\end{array}$ & 172 & 60,156 & 16 & 1904 & 146 & 53,553 & 212 & 143,371 \\
\hline $\begin{array}{l}\text { No. of } 1 \text { Dsq } \\
\text { reads } \\
\text { pass }\end{array}$ & 68 & 25,755 & 0 & 192 & 69 & 29,349 & 124 & 62,452 \\
\hline $\begin{array}{l}\text { No. of } 1 \text { Dsq } \\
\text { reads fail }\end{array}$ & 104 & 34,401 & 16 & 1712 & 77 & 24,204 & 88 & 80,919 \\
\hline $\begin{array}{l}\text { Average } \\
\text { length of } \\
\text { 1D reads }\end{array}$ & 5066.15 & 7195.29 & 3450.08 & 6484.00 & 5113.00 & 8270.88 & 6534.03 & 9417.60 \\
\hline $\begin{array}{l}\text { Longest 1D } \\
\text { read }\end{array}$ & 42,781 & 470,735 & 32,138 & 330,795 & 37,229 & $1,132,445$ & 56,642 & 485,807 \\
\hline $\begin{array}{l}\text { Average } \\
\text { length } \\
\text { of 1Dsq } \\
\text { reads }\end{array}$ & 5335.22 & 7685.61 & 2853.62 & 7344.74 & 5273.86 & 8472.84 & 5529.57 & 9829.82 \\
\hline $\begin{array}{l}\text { Longest } \\
\text { 1Dsq read }\end{array}$ & 18,489 & 43,102 & 6523 & 32,705 & 22,740 & 59,564 & 25,876 & 66,185 \\
\hline
\end{tabular}

Notes: "Pass" and "fail" refer to reads that met or did not meet the quality threshold, respectively. Run 2 was conducted on a previously used flow cell after $64-72 \mathrm{~h}$ run time and so had few pores left

Abbreviations: na, not applicable 
assembly with each of the three assemblers used: Abruijn, Canu and SMARTdenovo. All of the resulting assemblies that produced contiguous sequences were polished with Nanopolish. Eight rounds of Nanopolish polishing were performed on the Canu and SMARTdenovo assemblies and seven rounds were performed on the Abruijn assemblies (which get polished once by Abruijn).

All assemblies and polished versions of the assemblies were aligned to the corresponding reference genome using the LAST aligner (version 876) [27] following the example for human-ape alignments [28]. Briefly, the reference genome was indexed using LAST, then substitution and gap frequencies were determined using the last-train method [29]. Finally, alignments were performed using the lastal method and the determined substitution and gap frequencies. The resulting alignments were then filtered to retain only those alignments with an error probability $<1 \mathrm{e}^{-5}$. Giardia AWB assemblies were aligned to only the contigs from the reference genome labelled GLCHR01, GLCHR02, GLCHR03, GLCHR04 and GLCHR05 (representing the five chromosomes of G. intestinalis). Filtered alignments were converted to other file formats (for metric calculation) using the maf-convert method in the LAST aligner.

Average percent identity was calculated from alignments in blasttab format by taking the sum of the percent identity multiplied by the alignment length for each aligned portion and dividing that sum by the total alignment length. Proportion of mismatching bases was calculated from alignments in psl format by taking the sum of mismatching bases for all aligned portions divided by the total alignment length. Total number of indels per 1000 aligned bases was calculated from alignments in psl format by taking the sum of the number of insertions in the query and the number of insertions in the target for all aligned portions, dividing that sum by the total alignment length and multiplying by 1000. Average size of indels was calculated from alignments in psl format by taking the sum of the number of bases inserted in the query and the number of bases inserted in the target for all aligned portions and dividing that sum by the total number of indels. The proportions of the reference covered $0,1,2,3$ or 4 times were calculated using BEDtools (version v2.27.1) [30]. Alignments were first converted to SAM format and SAMtools was used to sort the alignment and convert it to a bam file. The genomecov function of BEDtools was then used to analyze the coverage of every base in the reference genome in the alignment. The proportion of bases in the reference genome with $0,1-, 2-, 3$ - and 4 -fold coverage in the assembly were retrieved.

The assembly evaluation metrics number of contigs and genome size were calculated for each assembly from the assembly fasta file. BUSCOs were calculated for each assembly using BUSCO v3.0.2 (BLAST+ v2.6.0, HMMER v3.1b2 and AUGUSTUS v3.2.3), with the eukaryote_odb9 dataset and default options (-sp fly) [31].

Average and standard deviation values for the groupings presented in the tables and figures for each metric were calculated in $\mathrm{R}$ [32]. $\mathrm{R}$ was also used to construct the scatter plots for the figures.

\section{Hybrid assemblies}

Hybrid genome assemblies were generated using the program Pilon (version 1.22) [33]. Briefly, short, highly accurate reads are mapped to a long-read assembly to correct for the higher error rate in the long reads. For each hybrid assembly, the Illumina reads were mapped using BWA to the long-read assembly. After sorting and indexing the alignments with SAMtools, pilon was run with default parameters to generate the hybrid assemblies.

The improvement of the hybrid assembly over the longread assembly from which it was built was measured by the BUSCO scores of each (calculated as described above). BUSCO scores were preferred because they do not depend on having a reference sequence and gene finding depends on assembly accuracy. The best hybrid assembly for each isolate was deposited at DDBJ/ENA/ GenBank under the accession numbers VSRS00000000 (Giardia beaver), VSRT00000000 (Giardia AWB) and VSRU00000000 (Giardia BGS). The versions described in this paper are versions VSRS01000000, VSRT01000000 and VSRU01000000, respectively.

\section{Draft annotation of hybrid genomes}

Gene models were transferred from the AWB reference genome to the hybrid genomes by mapping known proteins from the Giardia AWB reference genome to the hybrid assembly with the program exonerate v2.2.0 [34]. Only the best match for each query protein was retained in the annotation. The draft annotations can be found in Additional files 2, 3 and 4 for Giardia AWB, BGS and Beaver, respectively.

\section{Structural variant prediction and analysis}

Structural variants were predicted using the programs ngmlr and sniffles [12]. For each Giardia isolate, the long reads (without any correction) were mapped to the best hybrid assembly using ngmlr v0.2.7. The resulting alignments were sorted with SAMtools and the variants were called with sniffles v1.0.10.

Genes likely to be affected by the structural variants were identified by computing the overlapping regions 
between the genes found in the annotation step and the variant regions using BEDtools.

For each variant type, the list of putatively affected genes was examined, and genes of interest were analyzed for enrichment in the variants. For each predicted variant, 10,000 random samples of the same size as the variant were selected from the genome. For each sample the overlapping genes were found, and the genes of interest were counted. The 95th percentile was calculated from the resulting distribution of genes of interest using the nearest-rank method to find the count above which there is significant enrichment of the gene of interest (i.e. the cut-off for rejecting $\mathrm{H}_{0}$ ). The subsampling experiment was implemented in Java, the code for which is available on github at https://github.com/StephenMJPollo/ SV_Subsampling.

\section{Genome assembly for Giardia beaver}

The genome of Giardia beaver was assembled de novo the same as AWB and BGS hybrid assemblies described above (long-read assembly from $1 \mathrm{D}$ minION reads using SMARTdenovo, addition of Illumina reads to create final hybrid assembly).

\section{Results}

\section{Reference quality assemblies} Performance of ONT long reads

The MinION sequencing runs used here produced several hundred thousand reads each with the exception of Run2, which was a second run conducted on a previously used flow cell (Table 1). In addition to producing fewer reads, re-using the flow cell also resulted in lower proportions of reads passing the quality threshold during basecalling with $64 \%$ and $81 \%$ of $1 \mathrm{D}$ reads passing in Run2 compared to $90-98 \%$ of $1 \mathrm{D}$ reads passing in Runs 1, 3 and 4 (Table 1). NanoOK [26] analysis of read error profiles showed that reads from Run2 have lower aligned base identity, higher substitutions per 100 bases, and higher indels per 100 bases compared to the other runs (Table 2).

NanoOK analysis of 1D read error profiles for all runs indicated a $9-17 \%$ error rate in the regions of reads that aligned to the reference genome (Table 2, aligned base identity) and a $24-46 \%$ error rate across the entirety of reads that aligned to the reference genome (Table 2, overall base identity). The analysis also showed more deleted bases than inserted bases in the reads (Table 2). Average and maximum read lengths for all runs are presented in Table 1. Notably, the maximum 1D read length generated in the sequencing runs analyzed here was $1,132,445$ bases, though this read did not align to any Giardia reference genome nor did it have significant BLAST hits longer than $\sim 45 \mathrm{bp}$ in the $\mathrm{nr}$ database (data not shown). It is presumably a strand that got stuck but continued to generate (incorrect) sequence data.

Results of the long-read assemblies are provided in Additional file 5: Text S2 and Fig. 1, which shows the effects of 1D vs 1Dsq input reads, assembly program and number of genome-polishing iterations on BGS assemblies for four of the metrics.

\section{Hybrid assemblies}

Hybrid assemblies for Giardia AWB were created from every AWB long-read assembly in Additional file 6: Table S1. All of the AWB hybrid assemblies with the highest complete BUSCO score (117, Additional file 6: Table S2) were constructed from a SMARTdenovo longread assembly. For this reason and because of the performance of the long-read SMARTdenovo assemblies in general (see Additional file 5: Text S2, discussion of longread assemblies), the Giardia BGS and beaver hybrid assemblies were constructed from Illumina reads and the SMARTdenovo assemblies of the 1D MinION reads. The AWB hybrid assemblies outperformed their longread counterparts in all metrics measured (Additional file 6: Tables S1 and S2) and, for all three isolates, the hybrid assemblies had higher complete BUSCO scores than their corresponding long-read assembly. The best hybrid assembly for each isolate was selected for all further analysis on the basis of maximum complete BUSCO score (AWB_hybrid_106_0150015723312338_1dsma rtx0, BGS_hybrid_gs3-20-2019_22372244_1dsmartx0, Beaver_hybrid_107218_2309_1dsmartx0). For each of these assemblies, alignment to the AWB reference genome showed that the full chromosome was recovered for chromosomes 1-4 and the majority of chromosome 5 was also recovered (Fig. 2). Transfer of gene models from the AWB reference genome to each of the hybrids resulted in 9639, 7234, and 9647 transferred genes in the AWB, BGS and beaver hybrid genomes, respectively.

\section{Structural variant analysis}

We predicted structural variants from the long reads and hybrid assemblies to examine the variation between the four copies of each chromosome in the Giardia isolates sequenced. Giardia AWB, BGS and beaver had 392, 1860 and 483 variants, respectively (Table 3), which affect 2072, 4151 and 3423 genes, respectively. For each isolate, the full lists of predicted structural variants and genes affected by each variant can be found in Additional file 6: Tables S3-S5. Notably among the genes affected are known virulence factors including variant-specific surface proteins (VSP), 
Table 2 Read error profiles for Giardia AWB and Giardia BGS MinION sequencing runs

\begin{tabular}{lllllll}
\hline Error type/reads & AWB_0150 & AWB_0157 & AWB_2331 & AWB_2338 & BGS_2237 & BGS_2244 \\
\hline Proportion of reads counted (\%) & 87.55 & 83.56 & 28.04 & 52.61 & 12.62 & 77.47 \\
Overall base identity (\%) & 76.907 & 74.577 & 54.293 & 65.904 & 58.255 & 56.636 \\
Overall base identity error rate (\%) & 23.093 & 25.423 & 45.707 & 34.096 & 41.745 & 43.364 \\
Aligned base identity (\%) & 90.526 & 89.352 & 83.076 & 83.915 & 91.429 & 89.954 \\
Aligned base identity error rate (\%) & 9.474 & 10.648 & 16.924 & 16.085 & 8.571 & 10.046 \\
Identical bases per 100 & 80.430 & 78.338 & 71.024 & 71.597 & 80.855 & 78.834 \\
Inserted bases per 100 & 5.291 & 3.881 & 7.811 & 5.087 & 3.473 & 4.478 \\
Deleted bases per 100 & 5.860 & 8.450 & 6.758 & 9.592 & 8.105 & 7.886 \\
Substitutions per 100 & 8.415 & 9.334 & 14.406 & 13.725 & 7.569 & 8.801 \\
Mean insertion & 1.638 & 1.462 & 1.755 & 1.480 & 1.482 & 1.530 \\
Mean deletion & 1.621 & 1.787 & 1.591 & 1.788 & 1.848 & 1.898 \\
\hline
\end{tabular}

Notes: Using NanoOK [26], 1D reads were aligned to the corresponding reference genome and the error profiles of aligned reads were evaluated. NanoOK outputs read error profiles for each reference contig. To obtain overall error profiles for all reads, the values for each contig were multiplied by the proportion of total reads that aligned to that contig. The sum of these values for each error metric were scaled according to the proportion of total sequencing reads that were used for NanoOK's analysis

tenascins and high cysteine membrane proteins [35]. In AWB, BGS and beaver 39, 97 and 56 of the structural variants were found to have significantly more VSP than expected, respectively. Figure 3 shows alignments of the three hybrid genomes to the AWB reference genome with the predicted structural variants for each genome.

\section{Genome of Giardia beaver}

The genome of Giardia beaver was assembled into 8 contigs totalling $11,467,485$ bp (Table 4 ). It has a maximum contig length of $2.759 \mathrm{Mb}$ and an N50 of $1.965 \mathrm{Mb}$ (Table 4). One hundred thirteen complete BUSCOs were found out of 134 detected across the three Giardia isolates examined here (Table 4). Giardia beaver has $49.56 \%$ GC content, similar to values found for Giardia AWB (49.0\%) and other assemblage A isolates $(49.25 \%$ and $49.04 \%$; $[2,36])$.

Transfer of gene models from the AWB reference genome to the Giardia beaver hybrid genome resulted in 9647 transferred genes (Table 4), 3423 of which lie in predicted structural variant regions (Table 3 ). Roughly one third of the genome was found to be in structural variant regions, most of which are inversions, though the longest variants tend to be deletions and duplications (Table 3).

\section{Discussion}

Long-read assemblies and assemblers that lead to reference quality hybrid assemblies

Among the three assemblers tested, the SMARTdenovo assemblies for both Giardia AWB and BGS showed the lowest variability in all metrics except average indel size (Fig. 1, Additional file 7: Figures S1-S10). Moreover, the
SMARTdenovo assemblies had the highest average values for average percent identity, BUSCO score and proportion of reference covered $1 \times$ (where higher values indicate better performance) (Additional file 6: Table S1) and consistently strong performance in all metrics except average indel size (Fig. 1, Additional file 7: Figures S1S10). Despite thirteen of the top performing assemblies (8 AWB, 5 BGS) being Abruijn assemblies (Additional file 6: Table S6), plotting values for each metric showed Abruijn had the most variable performance (Additional file 6: Tables S7, S8, Additional file 7: Figures S1-S10). Canu assemblies generally performed somewhere between the SMARTdenovo and Abruijn assemblies (Additional file 6: Tables S7, S8).

Analysis of the 207 AWB and 108 BGS assemblies indicates that the optimal long-read only assembly pipeline for MinION sequenced Giardia is a SMARTdenovo assembly from 1D reads (either pooled or non-pooled input to reach sufficient genome coverage) followed by four or five rounds of polishing with Nanopolish (see Additional file 5: Text S2, Additional file 6: Tables S9-S15, for discussion of 1D vs 1Dsq input reads, pooling different sequencing runs for the same organism and number of rounds of genome polishing). However, it was the unpolished long-read assemblies that resulted in the best hybrid assemblies (1D read, SMARTdenovo assembled, no polishing with Nanopolish; Additional file 6: Table S2). Interestingly, the BGS assemblies are larger than the reference BGS assembly that was generated from 454 data [4], potentially due to the fragmented nature of the reference assembly. The AWB and BGS hybrid assemblies generated here have higher complete BUSCO scores than the available reference genomes (117 for both hybrids vs 114 AWB 


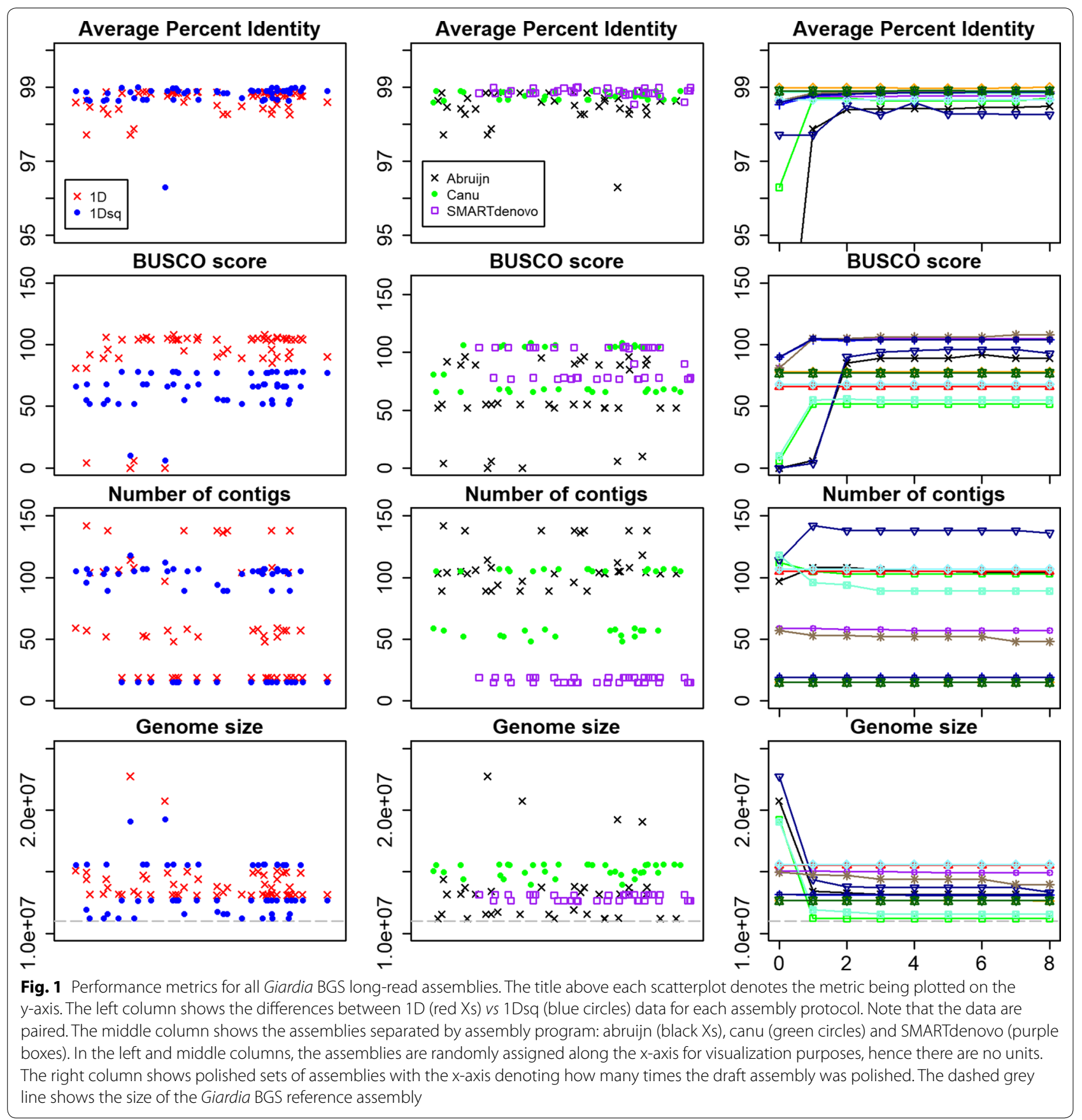

reference and 116 BGS reference) and were assembled into very large pieces (AWB hybrid N50: $616 \mathrm{~kb}$; BGS hybrid N50: $1645 \mathrm{~kb}$ ), suggesting they are of reference quality (Figs. 2, 3). Moreover, the hybrid genome for Giardia beaver has a similarly high complete BUSCO score and similar contig numbers and contig lengths to the AWB and BGS hybrids, indicating that reference quality assemblies can be generated de novo for Giardia with as little as one ONT MinION and one multiplexed Illumina MiSeq sequencing run. Most of the gene models from the AWB reference genome (9755) transferred to the hybrid genomes (Table 4), highlighting the completeness of the hybrid assemblies.

Each new release of a program specializing in handling long error prone reads can alter the optimal assembly pipeline for MinION data, but having the scripts to 


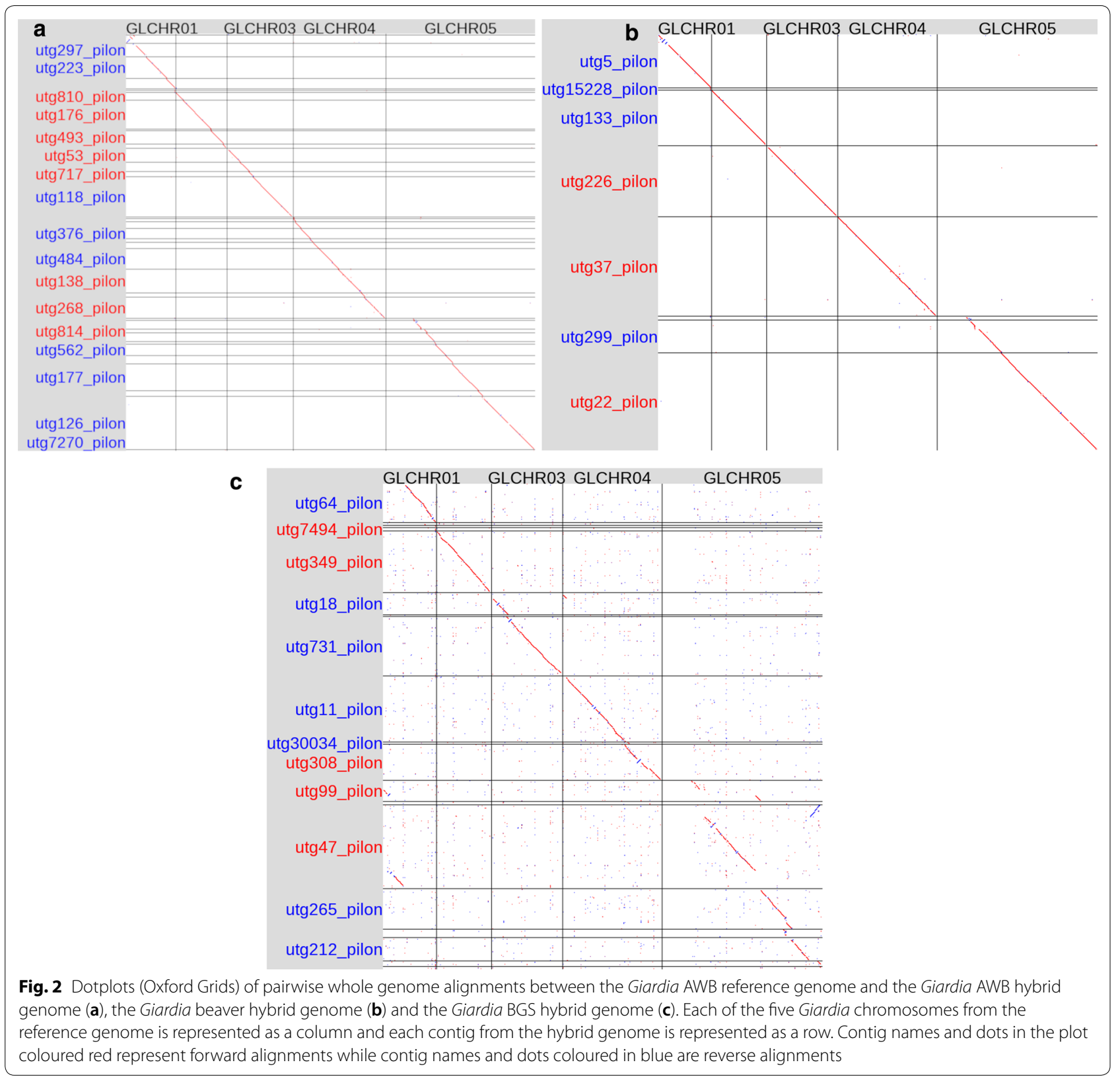

Table 3 Structural variants (SVs) in Giardia AWB, BGS and beaver

\begin{tabular}{llll}
\hline SV Property & AWB & BGS & Beaver \\
\hline No. of SVs & 392 & 1860 & 483 \\
No. of duplications & $45(14,520.4)$ & $185(48,239.6)$ & $69(37,535.0)$ \\
No. of deletions & $46(15,487.1)$ & $298(34,454.6)$ & $74(46,361.1)$ \\
No. of inversions & $162(19,437.9)$ & $746(28,782.2)$ & $234(12,866.7)$ \\
No. of inverted duplications & $2(2257.0)$ & $14(2680.1)$ & $0(0.0)$ \\
No. of transversions & $104(2.3)$ & $436(20.8)$ & $46(4.0)$ \\
No. of insertions & $33(299.6)$ & $181(596.4)$ & $60(286.9)$ \\
Proportion of genome contained in SVs & 0.1876 & 0.5662 & 0.3372 \\
No. of genes in SVs & 2072 & 4151 & 3423 \\
\hline
\end{tabular}




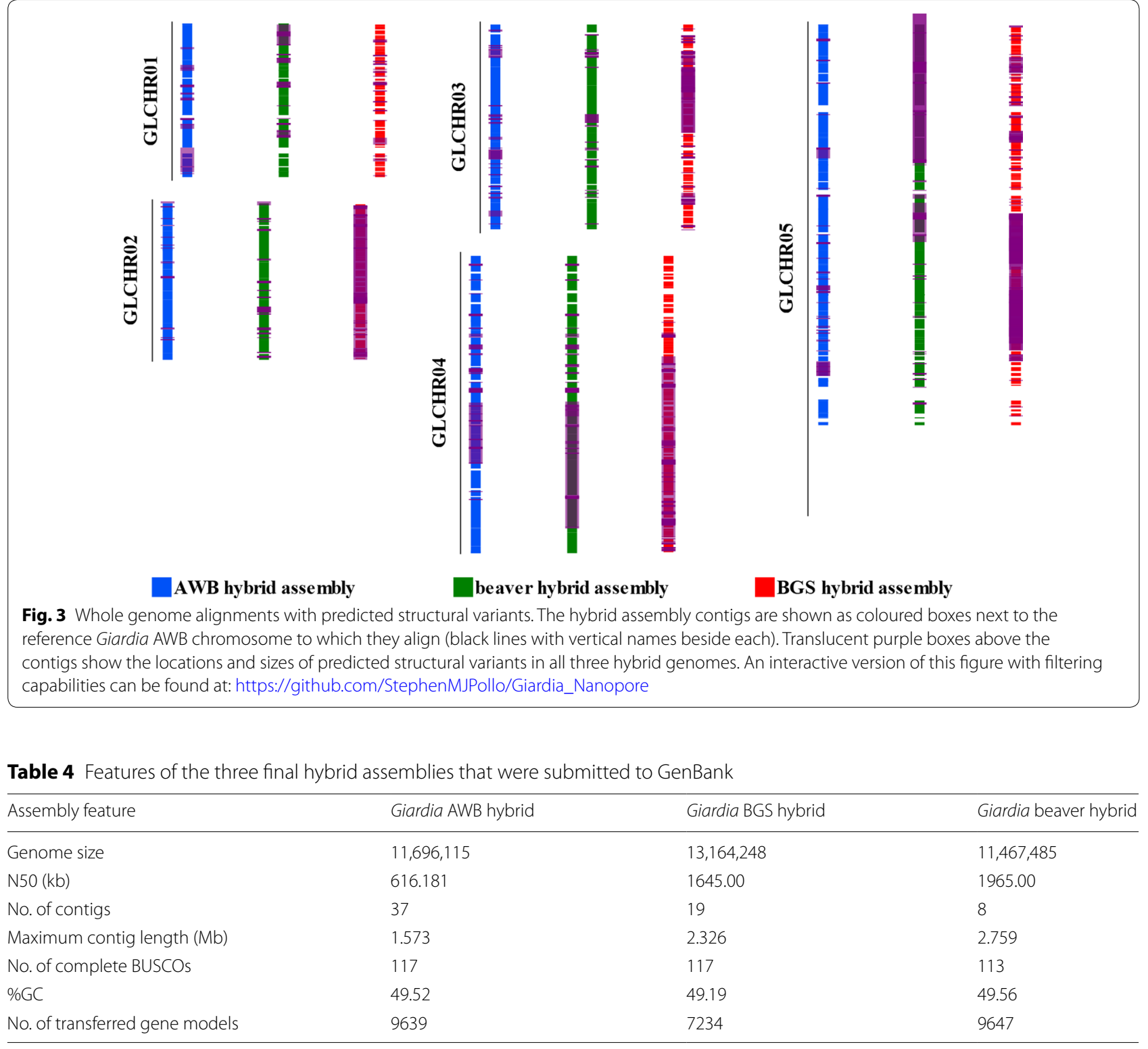

Table 4 Features of the three final hybrid assemblies that were submitted to GenBank

calculate the evaluation metrics used here enables rapid re-evaluations of assembler performance that could keep pace with software development. While the typical publication process, from numerous drafts of a manuscript and peer-review, cannot keep up with software development, a blog or community forum similar to an analysis on github of MinION basecalling programs [37] can and would therefore be more appropriate. These media also facilitate discussion on issues surrounding installation of programs and running them in various computing environments (e.g. some of the programs used here took up to a month to get installed and running properly). Combined with a current analysis of available long-read assemblers, such a forum would also allow researchers to determine which programs are worth the time to get working and when it may be a better use of time to go with programs that need less configuration (like Canu which worked immediately) but will still perform adequately for the intended purpose.

\section{Structural variants reveal different levels of intra-isolate variation}

Despite having similar genome sizes, the three isolates examined here have very different total numbers of variants detected and proportions of their genomes that are within a structural variant region (Table 3, Fig. 3). When 
Giardia BGS was first sequenced, the authors noted a much higher allelic sequence heterozygosity than what was observed in AWB (0.53\% in BGS vs $0.01 \%$ in AWB) [4]. The same trend is observed in the structural variants here with BGS being considerably more heterozygous than AWB. The differences in allelic sequence heterozygosity were attributed to AWB and BGS being in different assemblages [4]. While the values for Giardia beaver (an Assemblage A isolate) being more similar to AWB than BGS (Table 3) tentatively support the hypothesis that Assemblage B is more heterozygous than Assemblage A, many more genomes from each assemblage are needed to confirm it. Further, single cell sequencing could be used to examine the population structure of the isolates at a genetic level. Nonetheless, assemblage-specific variations in heterozygosity, or even isolate-specific variations in heterozygosity, will be important to consider in future comparisons between Giardia genomes. Previous genomic comparisons between assemblages [4] and within assemblages [38] have focused on SNPs and analyses of specific gene families. Including structural variant information provides a more complete picture of the heterozygosity and genetic diversity of each isolate by capturing differences in gene dosage as well as gene content.

\section{Effects of recombination in Giardia on structural variants}

Recombination between different cells (outcrossing) within and between isolates of Giardia has been suggested to occur through an as-yet undiscovered mechanism [39-42]. Outcrossing recombination events would allow for changes in gene copy number if the event involved or encompassed a structural variant like a duplication or deletion. Alternatively, large inversions can prevent recombination in the inverted areas [43], preventing gene flow during recombination events in Giardia. These regions are therefore important to keep in mind in future studies on recombination in Giardia as they may confound the analyses. Several dozen structural variants from each of the isolates examined here were found to be significantly enriched for VSP, supporting the suggestion that recombination is a potential source of VSP variation [44]. Expansions and contractions of this gene family through inheritance during outcrossing events of duplicated or deleted loci that affect VSP could be an important factor in the number and distribution of these genes between the various Giardia assemblages and isolates. As key surface proteins involved in host immune evasion [45], these expansions and contractions of the VSP repertoire could partially explain differences in pathogenicity between isolates. Moreover, as mediators of the Giardia cell's interaction with its surrounding environment, expansions and contractions of the VSP repertoire could affect host range. Alternatively, these genes could be hotspots for recombination events that generate structural variants. Then in addition to their roles as surface proteins they would also be potential factors influencing the evolution of Giardia genomes.

\section{Conclusions}

The present study demonstrates that high quality genomes can be generated for Giardia for a few thousand dollars per genome, thus enabling future large-scale comparative genomic studies of the genus. Moreover, thirdgeneration long reads can be further used to investigate heterozygosity and genome organization in Giardia despite its tetraploidy. We showed that structural variant regions affect many genes notably virulence factors including VSP, suggesting an important mechanism in the inheritance and distribution of these proteins among Giardia isolates. Finally, we have generated a reference genome sequence for a new isolate, Giardia beaver, with accompanying prediction of its structural variants.

\section{Supplementary information}

Supplementary information accompanies this paper at https://doi. org/10.1186/s13071-020-3968-8.

Additional file 1: Text S1. Plaintext of commands used in analyses. Additional file 2: Draft annotation (gff format) of AWB hybrid genome. Additional file 3: Draft annotation (gff format) of BGS hybrid genome. Additional file 4. Draft annotation (gff format) of beaver hybrid genome. Additional file 5: Text S2. Additional results and discussion on long-read assemblies.

Additional file 6: Table S1. All metrics for AWB and BGS long-read assemblies. Table S2. All metrics for AWB hybrid assemblies. Table S3. Overlapping structural variants and genes in AWB. Table S4. Overlapping structural variants and genes in BGS. Table S5. Overlapping structural variants and genes in Giardia beaver. Table S6. Top performing AWB and BGS long-read assemblies. Table S7. Summarized assembler metrics in AWB long-read assemblies. Table S8. Summarized assembler metrics in BGS long-read assemblies. Table S9. Summarized metrics for 1D vs 1Dsq AWB long-read assemblies. Table S10. Summarized metrics for 1D vs 1Dsq BGS long-read assemblies. Table S11. All metrics for corresponding 1D and 1Dsq AWB and BGS long-read assemblies. Table S12. Summarized metrics for pooling input AWB long-read assemblies. Table S13. Summarized metrics for pooling input BGS long-read assemblies. Table S14. Summarized metrics for polishing AWB long-read assemblies. Table S15. Summarized metrics for polishing BGS long-read assemblies.

Additional file 7: Figure S1. 1D and 1Dsq AWB long-read assembly performance. Figure S2. Corresponding 1D and 1Dsq AWB long-read assembly performance. Figure S3. AWB long-read assembly performance by assembly program. Figure S4. Pooling input AWB long-read assembly performance. Figure S5. Performance of polishing AWB long-read assemblies. Figure S6. 1D and 1Dsq BGS long-read assembly performance. Figure S7. Corresponding 1D and 1Dsq BGS long-read assembly performance. Figure S8. BGS long-read assembly performance by assembly program. Figure S9. Pooling input BGS long-read assembly performance. Figure S10. Performance of polishing BGS long-read assemblies. 


\section{Abbreviations}

BUSCO: benchmarking universal single copy orthologs; ONT: Oxford Nanopore Technologies; SNPs: single nucleotide polymorphisms; SRA: sequence read archive; SVs: structural variants; VSP: variant-specific surface proteins.

\section{Acknowledgements}

The authors would like to thank Chanwoori Jeong (Faculty of Veterinary Medicine, University of Calgary) for drawing the graphical abstract for this paper.

\section{Authors' contributions}

GWB, JW and SJR were in charge of resources and investigation. SMJP carried out investigation, formal analysis, software, writing original draft and visualization. RAG, JY, BRD and JDW carried out funding acquisition and supervision. SMJP, SJR, MLW, RAG, BRD and JDW were involved in conceptualization and methodology. All authors read and approved the final manuscript.

\section{Funding}

This study was supported by the Ontario Ministry of Agriculture, Food and Rural Affairs (OMAFRA \#FS2016-3010) to BRD, Alberta Agriculture and Forestry (AAF \#2016F013R) to JDW, Natural Sciences and Engineering Research Council of Canada (NSERC) Discovery (\#222982) to JY and a NSERC Visiting Fellowship in Canadian Government Laboratories to SJR. None of the funding agencies had any role in the design of the study, the collection, analysis and interpretation of data, nor in the writing of the manuscript.

\section{Availability of data and materials}

The datasets generated and analysed during the present study are available in the SRA under the accession number PRJNA561185. The hybrid assemblies generated are available from GenBank under the accession numbers VSRS00000000 (Giardia beaver), VSRT00000000 (Giardia AWB) and VSRU00000000 (Giardia BGS). The versions described in this paper are versions VSRS01000000, VSRT01000000 and VSRU01000000, respectively. All other supporting materials are included in this published article and its additional files and can also be found at https://github.com/StephenMJPollo/Giardia_Nanop ore. The software that runs the genome sampling can be found at https:// github.com/StephenMJPollo/SV_Subsampling (Project name: SV_Subsampling; Project home page: https://github.com/StephenMJPollo/SV_Subsa mpling; Archived version: https://doi.org/10.5281/zenodo.3445450; Operating system: Linux; Programming language: Java; Other requirements: BEDtools. License: GNU GPLV3)

\section{Ethics approval and consent to participate}

Not applicable.

\section{Consent for publication}

Not applicable.

\section{Competing interests}

The authors declare that they have no competing interests.

\section{Author details}

${ }^{1}$ Department of Ecosystem and Public Health, Faculty of Veterinary Medicine, University of Calgary, Calgary, AB, Canada. ${ }^{2}$ Host-Parasite Interactions Training Program, University of Calgary, Calgary, AB, Canada. ${ }^{3}$ Bureau of Microbial Hazards, Food Directorate, Health Canada, Ottawa, ON, Canada. ${ }^{4}$ Department of Comparative Biology and Experimental Medicine, Faculty of Veterinary Medicine, University of Calgary, Calgary, AB, Canada. ${ }^{5}$ Division of Enteric Diseases, National Microbiology Laboratory, Public Health Agency of Canada Guelph, ON, Canada. ${ }^{6}$ Department of Biology, Biochemistry and Molecular Biology Program, Trent University, Peterborough, ON, Canada.

Received: 3 October 2019 Accepted: 13 February 2020 Published online: 28 February 2020

\section{References}

1. Certad G, Viscogliosi E, Chabé M, Cacciò SM. Pathogenic mechanisms of Cryptosporidium and Giardia. Trends Parasitol. 2017;33:561-76.
2. Morrison HG, McArthur AG, Gillin FD, Aley SB, Adam RD, Olsen GJ, et al. Genomic minimalism in the early diverging intestinal parasite Giardia lamblia. Science. 2007:317:1921-6.

3. Aurrecoechea C, Brestelli J, Brunk BP, Carlton JM, Dommer J, Fischer $\mathrm{S}$, et al. GiardiaDB and TrichDB: integrated genomic resources for the eukaryotic protist pathogens Giardia lamblia and Trichomonas vaginalis. Nucleic Acids Res. 2009:37:526-30.

4. Franzén O, Jerlström-Hultqvist J, Castro E, Sherwood E, Ankarklev J, Reiner DS, et al. Draft genome sequencing of Giardia intestinalis Assemblage $B$ isolate GS: is human giardiasis caused by two different species? PLoS Pathog. 2009;5:e1000560.

5. Stroehlein AJ, Korhonen PK, Chong TM, Lim YL, Chan KG, Webster B, et al. High-quality Schistosoma haematobium genome achieved by singlemolecule and long-range sequencing. Gigascience. 2019;8:giz108.

6. Liechti N, Schürch N, Bruggmann R, Wittwer M. Nanopore sequencing improves the draft genome of the human pathogenic amoeba Naegleria fowleri. Sci Rep. 2019;9:16040.

7. Díaz-Viraqué F, Pita S, Greif G, de Souza RCM, Iraola G, Robello C Nanopore sequencing significantly improves genome assembly of the protozoan parasite Trypanosoma cruzi. Genome Biol Evol. 2019;11:1952-7.

8. Rhoads A, Au KF. PacBio sequencing and its applications. Genom Proteom Bioinform. 2015;13:278-89.

9. Tyson JR, O'Neil NJ, Jain M, Olsen HE, Hieter P, Snutch TP. MinlON-based long-read sequencing and assembly extends the Caenorhabditis elegans reference genome. Genome Res. 2018;28:266-74.

10. Lu H, Giordano F, Ning Z. Oxford nanopore minION sequencing and genome assembly. Genom Proteom Bioinform. 2016;14:265-79.

11. de Koning APJ, Gu W, Castoe TA, Batzer MA, Pollock DD. Repetitive elements may comprise over two-thirds of the human genome. PLoS Genet. 2011;7:e1002384.

12. Sedlazeck FJ, Rescheneder P, Smolka M, Fang H, Nattestad M, Von Haeseler $A$, et al. Accurate detection of complex structural variations using single-molecule sequencing. Nat Methods. 2018;15:461-8.

13. Jeffares DC, Jolly C, Hoti M, Speed D, Shaw L, Rallis C, et al. Transient struCtural variations have strong effects on quantitative traits and reproductive isolation in fission yeast. Nat Commun. 2017:8:14061.

14. Weischenfeldt J, Symmons O, Spitz F, Korbel JO. Phenotypic impact of genomic structural variation: insights from and for human disease. Nat Rev Genet. 2013;14:125-38.

15. Kyriakidou M, Tai HH, Anglin NL, Ellis D, Strömvik MV . Current strategies of polyploid plant genome sequence assembly. Front Plant Sci. 2018;9:1660.

16. Feng Y, Zhang Y, Ying C, Wang D, Du C. Nanopore-based fourthgeneration DNA sequencing technology. Genom Proteom Bioinform. 2015;13:4-16.

17. Clark CG, Diamond LS. Methods for cultivation of luminal parasitic protists of clinical importance. Clin Microbiol Rev. 2002;15:329-41.

18. Vera D. Dockerfile for the Albacore basecaller from Oxford Nanopore. 2017. https://github.com/dvera/albacore. Accessed 1 Mar 2017.

19. Lin Y, Yuan J, Kolmogorov M, Shen MW, Chaisson M, Pevzner PA. Assembly of long error-prone reads using de Bruijn graphs. Proc Natl Acad Sci USA. 2016:113:E8396-405

20. Koren S, Walenz BP, Berlin K, Miller JR, Bergman NH, Phillippy AM. Canu: scalable and accurate long-read assembly via adaptive $k$-mer weighting and repeat separation. Genome Res. 2017;27:722-36.

21. Ruan J. Ultra-fast de novo assembler using long noisy reads. 2017. https:// github.com/ruanjue/smartdenovo. Accessed 1 Mar 2017.

22. Loman NJ, Quick J, Simpson JT. A complete bacterial genome assembled de novo using only nanopore sequencing data. Nat Methods. 2015;12:733-5.

23. Simpson J. Signal-level algorithms for MinION data. 2017. https://githu b.com/jts/nanopolish. Accessed 1 Mar 2017

24. Li H, Durbin R. Fast and accurate long-read alignment with BurrowsWheeler transform. Bioinformatics. 2010;26:589-95.

25. Cock PJA, Bonfield JK, Chevreux B, Li H. SAM/BAM format v1.5 extensions for de novo assemblies. bioRxiv. 2015. https://doi.org/10.1101/020024.

26. Leggett RM, Heavens D, Caccamo M, Clark MD, Davey RP. NanoOK: multireference alignment analysis of nanopore sequencing data, quality and error profiles. Bioinformatics. 2016;32:142-4.

27. Kielbasa SM, Wan R, Sato K, Horton P, Frith MC. Adaptive seeds tame genomic sequence comparison. Genome Res. 2011;21:487-93. 
28. Mcfrith/last-genome-alignments. 2017. https://github.com/mcfrith/lastgenome-alignments. Accessed 1 Mar 2017.

29. Hamada M, Ono Y, Asai K, Frith MC. Training alignment parameters for arbitrary sequencers with LAST-TRAIN. Bioinformatics. 2017;33:926-8.

30. Quinlan AR, Hall IM. BEDTools: a flexible suite of utilities for comparing genomic features. Bioinformatics. 2010;26:841-2.

31. Simão FA, Waterhouse RM, loannidis P, Kriventseva EV, Zdobnov EM. BUSCO: assessing genome assembly and annotation completeness with single-copy orthologs. Bioinformatics. 2015;31:3210-2.

32. R Core Team. R: A language and environment for statistical computing. Vienna, Austria: R Foundation for Statistical Computing; 2013. http:// www.r-project.org/.

33. Walker BJ, Abeel T, Shea T, Priest M, Abouelliel A, Sakthikumar S, et al. Pilon: an integrated tool for comprehensive microbial variant detection and genome assembly improvement. PLOS ONE. 2014;9:e112963.

34. Slater GSC, Birney E. Automated generation of heuristics for biological sequence comparison. BMC Bioinform. 2005;6:31.

35. Dubourg A, Xia D, Winpenny JP, Al Naimi S, Bouzid M, Sexton DW, et al. Giardia secretome highlights secreted tenascins as a key component of pathogenesis. Gigascience. 2018;7:1-13.

36. Adam RD, Dahlstrom EW, Martens CA, Bruno DP, Barbian KD, Ricklefs SM, et al. Genome sequencing of Giardia lamblia genotypes A2 and B isolates (DH and GS) and comparative analysis with the genomes of genotypes A1 and E (WB and Pig). Genome Biol Evol. 2013;5:2498-511.

37. Wick R. A comparison of different Oxford nanopore basecallers. 2017. https://github.com/rrwick/Basecalling-comparison\#m. Accessed 1 Mar 2017.

38. Ankarklev J, Franzén O, Peirasmaki D, Jerlström-Hultqvist J, Lebbad M, Andersson J, et al. Comparative genomic analyses of freshly isolated Giardia intestinalis assemblage A isolates. BMC Genom. 2015;16:697.
39. Cooper MA, Sterling CR, Gilman RH, Cama V, Ortega Y, Adam RD. Molecular analysis of household transmission of Giardia lamblia in a region of high endemicity in Peru. J Infect Dis. 2010;202:1713-21.

40. Cooper MA, Adam RD, Worobey M, Sterling CR. Population genetics provides evidence for recombination in Giardia. Curr Biol. 2007;17:1984-8.

41. Ankarklev J, Lebbad M, Einarsson E, Franzén O, Ahola H, Troell K, et al. A novel high-resolution multilocus sequence typing of Giardia intestinalis assemblage $A$ isolates reveals zoonotic transmission, clonal outbreaks and recombination. Infect Genet Evol. 2018;60:7-16.

42. Birky CW. Giardia sex? Yes, but how and how much? Trends Parasitol. 2010:26:70-4.

43. Wellenreuther M, Mérot C, Berdan E, Bernatchez L. Going beyond SNPS: the role of structural genomic variants in adaptive evolution and species diversification. Mol Ecol. 2019;28:1203-9.

44. Jerlström-Hultqvist J, Franzén O, Ankarklev J, Xu F, Nohýnková E, Andersson JO, et al. Genome analysis and comparative genomics of a Giardia intestinalis assemblage E isolate. BMC Genom. 2010;11:543.

45. Prucca CG, Slavin I, Quiroga R, Elías EV, Rivero FD, Saura A, et al. Antigenic variation in Giardia lamblia is regulated by RNA interference. Nature. 2008;456:750-4.

\section{Publisher's Note}

Springer Nature remains neutral with regard to jurisdictional claims in published maps and institutional affiliations. 